\title{
Diagnostic Efficacy of MRI in the Evaluation of Cerebellopontine Angle Tumors
}

\author{
Amritha $A^{1}$, Abubacker Sulaiman $F^{2}$, E.A. Parthasarathy ${ }^{3}$, Ramya Kalaiarasan ${ }^{4}$, Rajkumar $\mathbf{G}^{5}$, Farooque M.C ${ }^{6}$ \\ ${ }^{1}$ Final Year Postgraduate, Department of Radiology, Chettinad Hospital and Research Institute, ${ }^{2}$ Professor, Department of \\ Radiology, Chettinad Hospital and Research Institute, ${ }^{3}$ Assistant Professor, Department of Radiology, Chettinad Hospital and \\ Research Institute, ${ }^{4}$ Senior Resident, Department of Radiology, Chettinad Hospital and Research Institute, ${ }^{5}$ Senior Resident, \\ Department of Radiology, Chettinad Hospital and Research Institute, ${ }^{6}$ Senior Resident, Department of Radiology, Chettinad \\ Hospital and Research Institute, India
}

Corresponding author: Dr. Abubacker Sulaiman F, Professor, Department of Radiology, Chettinad Hospital and Research Institute, Rajiv Gandhi Salai, Kelambakkam, Chennai, Tamilnadu, India

DOI: http://dx.doi.org/10.21276/ijcmsr.2019.4.3.14

How to cite this article: Amritha A, Abubacker Sulaiman F, E.A. Parthasarathy, Ramya Kalaiarasan, Rajkumar G, Farooque M.C. Diagnostic efficacy of MRI in the evaluation of cerebellopontine angle tumors. International Journal of Contemporary Medicine Surgery and Radiology. 2019;4(3):C63-C68.

\section{A B S T R A C T}

Introduction: Magnetic Resonance Imaging (MRI) has been the primary imaging modality and has revolutionized the imaging of brain tumours. MRI can display accurate multi planer imaging without interfering of adjacent structures especially for posterior fossa mass lesion. MRI is the imaging modality of choice for cerebollo-pontine (CP) angle Schwannoma. The aim of the current study was to assess the effectiveness of MRI in the evaluation of cerebellopontine angle lesions, with histopathology as gold standard in comparison and also to estimate the accuracy, sensitivity, specificity of MRI in the detection of cerebellopontine angle lesions.

Material and Methods: This was a retrospective study which comprised of 50 patients with clinical suspicion of cerebellopontine angle lesions. The study duration was from June 2011 to March 2018 for imaging. Patients presented with signs and symptoms of any lesion in the CP angle were included in the study. Patients excluded from the study were those with contraindications to MRI and with history of previous surgical intervention.

Results: In the present study, 22 were males and 28 were females. The age group of the patients ranged between 8 to 72 years. Majority of our patients were aged between 51 - 60 years. Out of 50 patients, 22 patients had acoustic schwannoma on MRI, 14 patients were diagnosed to have cerebellopontine angle meningioma, 8 patients had epidermoid cyst, 4 patients had arachnoid cyst and 2 patients had lipoma and metastasis respectively.

Conclusion: MR imaging is the study of choice for the examination of the patient of cerebellopontine angle Schwannoma because of its high sensitivity especially after use of contrast material.

Keywords: Acoustic Schwannoma, MRI, Cerebellopontie Angle, Meningioma

\section{INTRODUCTION}

A schwannoma is a tumor that arises from Schwann cells, named after their discoverer, Theodor Schwann (a 19th Century German histologist and physiologist). Schwann cells are cells that are derived from a primitive structure known as the neural crest. Schwann cells, like the central nervous system's oligodendrocytes, lay down the myelin sheath. Theoretically, schwannomas can arise anywhere that a Schwann cell is found. However, in the brain and spinal cord, schwannomas tend to arise mainly at the root entry zones. When the VIII ${ }^{\text {th }}$ (vestibulocochlear) cranial nerveis involved where they are referred to as vestibular Schwannomas (or acoustic "neuromas"). When the Vth (trigeminal) cranial nerve is involved it is referred to as trigeminal Schwannomas. When the lower cranial nerves (IX, X or XI-i.e., glossopharyngeal, vagus, or accessory) at the jugular foramen is involved it is referred to as jugular foramen Schwannomas. The spinal cord's spinal nerve roots (especially sensory or "dorsal" nerve roots)-where they are referred to as spinal Schwannomas. .,2,3 $^{1}$

The cerebellopontine (CPA) lesion is a cerebrospinal fluid (CSF) filled subarachnoid space that contains cranial nerves and vessels. The CPA is bounded by the pons, anterior aspect of the cerebellum and the petrous temporal bone covered by dura mater. The cerebellopontine angle (CPA) is the most common site for posterior fossa neoplasms. Tumours occupying this region account for approximately $10 \%$ of all intracranial neoplasms, with vestibular schwannomas accounting for $80 \%$ of these CPA tumours. Other tumours involving this region include meningiomas, dermoid tumours, arachnoid cysts, lipomas, and metastases. The cerebellopontine angle is densely inhabited by vital neurologic tissue. As such, tumour growth in this region may give rise to significant neurologic dysfunction and, if left untreated, can ultimately lead to death. ${ }^{4,5,6}$ 
CPA tumours, although uniform in location, are diverse pathologically and present clinically depending upon the site of tumour origin and displacement of the neurovascular structures. The tumours can derive from many anatomical structures, including primary origin from internal auditory meatus, ponto-cerebellar cistern, and the lateral recess of the fourth ventricle, temporal bone, brain stem, or cerebellar nervous tissue. Clinical presentation of the CPA tumours is variable and it depends upon the size and location of the tumour. It can be asymptomatic in early stage or it can give vertigo, tinnitus, or hearing loss. ${ }^{7,8}$

There is requirement of the knowledge about the anatomy, pathology, clinical presentations and merits of different imaging modalities can lead to adequate diagnosis and management of the different CPA lesions. CT and MRI are widely used radiological methods for CPA imaging. The main radiological diagnostic goal is the description of the relation of the tumour to IAM, the brain stem and cerebellar hemispheres. The second line basic information is if the lesion is extra- or intracerebral. ${ }^{9,10}$

Since the introduction of Magnetic Resonance (MR), malformations of the brain have been found with greater frequency than previously suspected. This is especially true for any lesions in the cerebellopontine angle, where the sensitivity and specificity of MR imaging with its multidimensional imaging capabilities are far superior to that of CT. The higher contrast, resolution and multi planar capabilities of MR helps to evaluate shape and margins, extent, mass effect, intensity at MR imaging, enhancement and adjacent bone reaction. The MR imaging technique is simple and non-invasive. ${ }^{11,12}$

\begin{tabular}{|l|c|c|}
\hline Type of lesion & Number of cases & Percentage \\
\hline Acoustic schwannoma & 22 & $44 \%$ \\
\hline Meningioma & 14 & $28 \%$ \\
\hline Epidermoid cyst & 8 & $16 \%$ \\
\hline Arachnoid cyst & 3 & $6 \%$ \\
\hline Others & 3 & $6 \%$ \\
\hline Total & 50 & 100 \\
\hline Table-1: Shows the distribution of type of lesions among study \\
subjects \\
\hline
\end{tabular}

\begin{tabular}{|l|c|c|}
\hline Symptoms & No. of patients & $\%$ \\
\hline Vertigo & 8 & $16 \%$ \\
\hline Tinnitus & 4 & $8 \%$ \\
\hline Headache & 16 & $32 \%$ \\
\hline Facial nerve palsy & 1 & $2 \%$ \\
\hline Vomiting & 8 & $16 \%$ \\
\hline Hearing abnormalities & 13 & $26 \%$ \\
\hline \multicolumn{2}{|c|}{ Table-2: Shows the distribution of symptoms among study } \\
subjects \\
\hline
\end{tabular}

Therefore, the aim of this study was to assess the effectiveness of MRI in the evaluation of cerebellopontine angle lesions, with histopathology as gold standard in comparison and also to estimate the accuracy, sensitivity, specificity of MRI in the detection of cerebellopontine angle lesions.

\section{MATERIAL AND METHODS}

This was a retrospective study which comprised of 50 patients with clinical suspicion of cerebellopontine angle lesions, which were referred to the Department of Radiodiagnosis Chettinad Hospital and Research Institute, from the period of June 2011 to March 2018 for imaging. Informed consent was taken from all patients undergoing this study. MRI was performed on GE Signa HDxt MR Scanner using standard imaging coil.

The most common symptoms associated with vestibular schwannomas were those caused by pressure on the cochlear and vestibular divisions of the eighth cranial nerve, namely, sensorineural hearing loss, tinnitus, and disequilibrium. Also they have difficulty understanding speech in the affected ear. The symptoms are usually slowly progressive, evolving over months or years. The larger tumours are more likely to cause trigeminal manifestations such as facial numbness and loss of the corneal reflex. Patients presented with signs and symptoms of any lesion in the CP angle were included in the study. Patients excluded from the study were those with contraindications to MRI and with history of previous surgical intervention.

The standard protocol consisted of T1WI, T2WI, DWI and FLAIR images in axial, sagittal and coronal planes. Each MR image was analysed for specific features that were relevant to the evaluation of cerebellopontine angle schwannoma.

\section{STATISTICAL ANALYSIS}

The data was tabulated into MS excel sheet and the analysis was performed using SPSS software. The descriptive summary of variables are presented through percentages as well as aided by appropriate graphs.

\section{RESULTS}

Table no. 1 shows the distribution of lesions among study subjects. It was found that acoustic schwannoma was found in maximum number of subjects (44\%) and least lesion which was found is arachnoid cyst (6\%) followed by meningioma (28\%) and epidermoid cyst (16\%). In the present study, Table no. 2 shows the distribution of symptoms and found that headache (32\%) was most commonly found among study subjects followed by hearing abnormalities (26\%), vomiting $(16 \%)$ and vertigo (16\%). The least symptoms found were facial nerve palsy (2\%) among study subjects.

In the current study, Table no. 3 shows that out of all the cases,

\begin{tabular}{|c|c|c|c|c|}
\hline \multicolumn{2}{|c|}{ Test Result MRI } & \multicolumn{2}{|l|}{ HPE } & \multirow{3}{*}{$\begin{array}{c}\text { Total } \\
45 \text { (TP+FP) }\end{array}$} \\
\hline & & \multirow{2}{*}{$\begin{array}{l}\text { Present Schwannoma } \\
\text { (44) True positive (TP) }\end{array}$} & \multirow{2}{*}{$\begin{array}{l}\text { Absent Schwannoma } \\
\text { (1) False positive (FP) }\end{array}$} & \\
\hline MRI & Positive & & & \\
\hline & Negative & 2 False negative (FN) & 3 True negative (TN) & $5(\mathrm{FN}+\mathrm{TN})$ \\
\hline \multicolumn{2}{|c|}{ Total } & 46 All patients with true Schwannoma (TP+FN) & 4 False Schwannoma (FP+TN) & $50(\mathrm{TP}+\mathrm{FP}+\mathrm{FN}+\mathrm{TN})$ \\
\hline
\end{tabular}



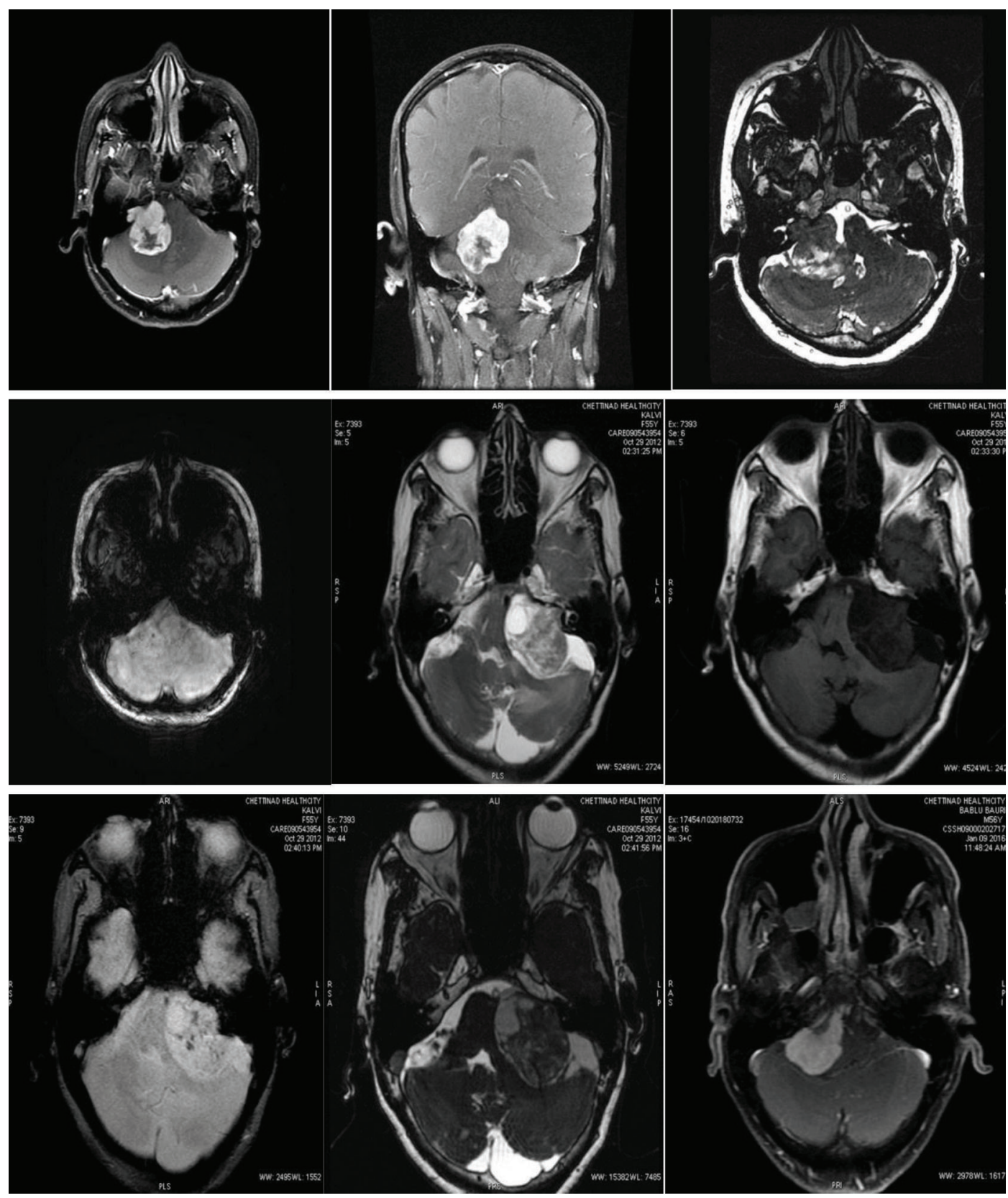

Figure-1: Shows MRI images of various types of Schwannomas among study subjects

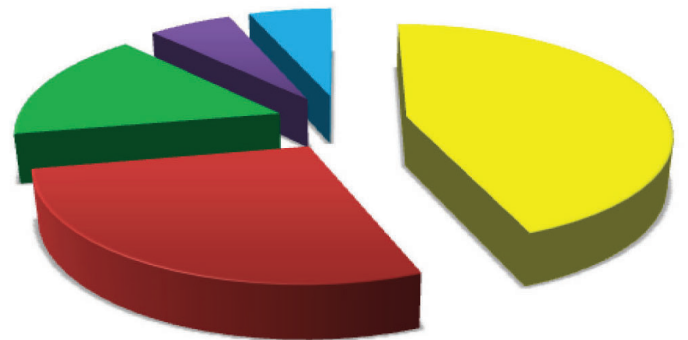

$\square$ Acoustic schwannoma Meningioma $\square$ Epidermoid cyst arachnoid cyst O Others

Graph-1: Shows the distribution of lesions in CP angle among study subjects

46 were true schwannomas and 4 were false schwannoma which were found to be true positive and false positive diagnosed by MRI and is confirmed by histopathological evaluation. Table no. 4 shows the sensitivity and specificity

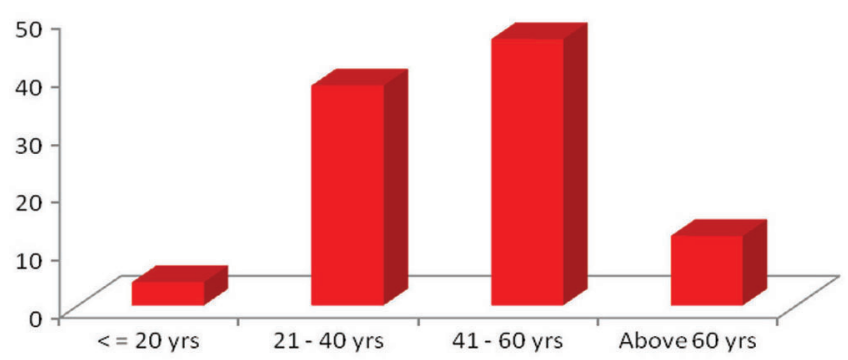

Graph-2: Shows the distribution of age among study subjects

of about $95 \%$ and $75 \%$ with positive and negative predictive value of $97 \%$ and $60 \%$ with accuracy rate of $85 \%$ with the help of MRI for diagnosis of schwannomas among study subjects.

Graph no. 1 shows the distribution of lesions in CP angle and found that Acoustic schwannoma were found to be among majority of the subjects followed by meningioma, epidermoid 

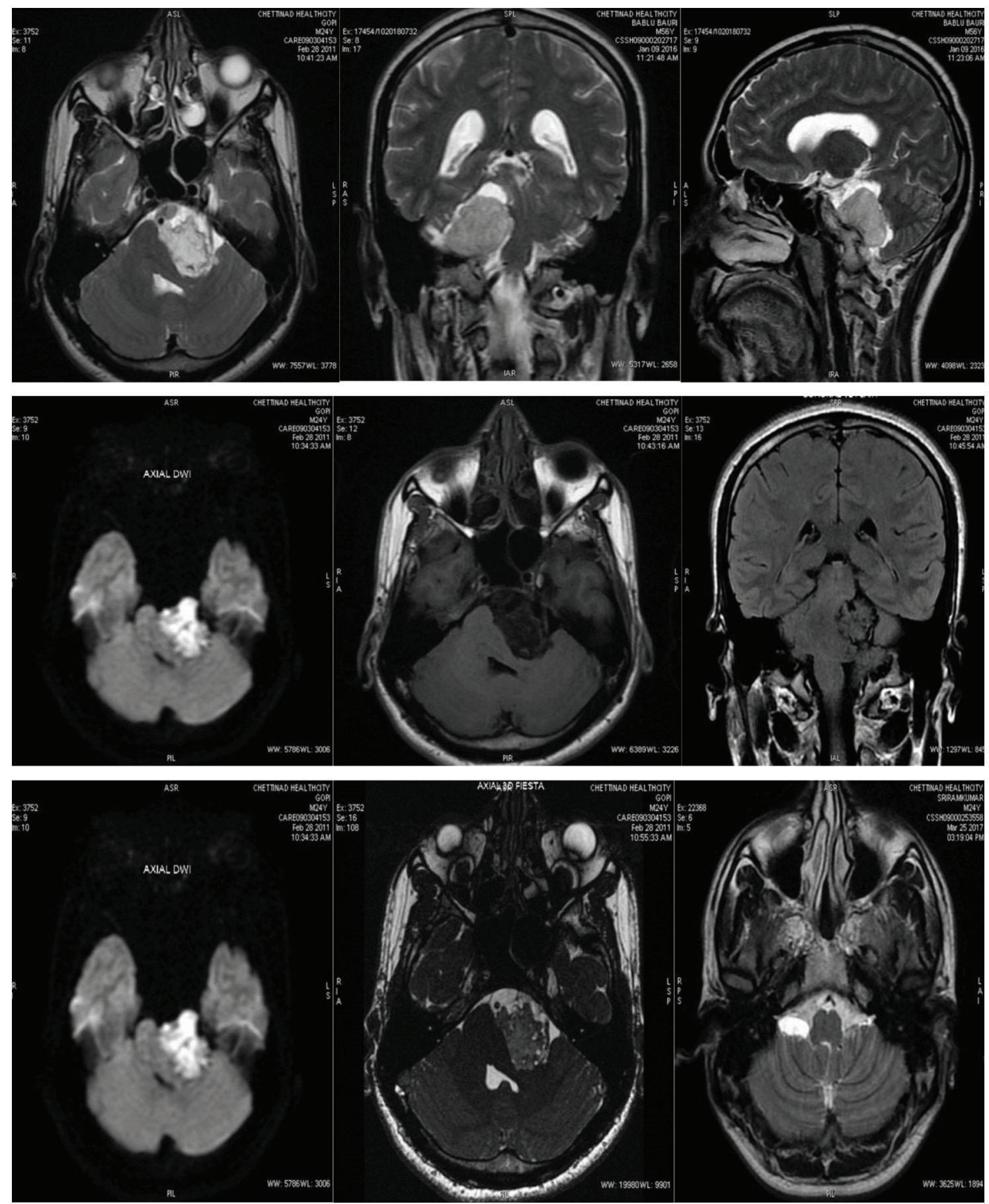

Figure-2: Shows images of histopathological findings of meningioma, arachnoid cyst, epidermoid cyst and lipoma among study subjects

\begin{tabular}{|l|c|}
\hline Sensitivity & $95.65 \%$ \\
\hline Specificity & $75.00 \%$ \\
\hline Positive predictive value & $97.78 \%$ \\
\hline Negative predictive value & $60.00 \%$ \\
\hline Accuracy & $85.33 \%$ \\
\hline \multicolumn{2}{|c|}{ Table-4: Shows sensitivity and specificity of MRI diagnosis } \\
among study subjects
\end{tabular}

and arachnoid cyst. Graph no. 2 shows the age distribution among study subjects and revealed that $41-60$ years of age were maximum followed by 21-40 years of age and above 60 years. Least number of subjects was found under the age group of 20 years.

Figure no. 1 shows MRI images of various types of schwannomas among study subjects. A well-defined lesion seen in the left CPA with extension into left internal acoustic meatus. A right cerebellopontine angle mass is intense to grey matter in T1 and T2 weighted images of meningioma. Figure no. 2 shows images of histopathological findings of meningioma, arachnoid cyst, epidermoid cyst and lipoma among study subjects.

\section{DISCUSSION}

Magnetic resonance imaging is a non-invasive and safe 
imaging modality. Nowadays it plays an excellent role in the detection of a spectrum of usual and unusual lesions and remains as a major imaging technique for detection and localization of cerebellopontine angle lesions. CPA pathologies can be asymptomatic or it may have varied presentation. Site of tumour origin is our primary concern. Other factors that are helpful in establishing the diagnosis include signal intensity at MR imaging, degree of enhancement, shape and margins, extent, mass effect and displacement of adjacent neurovascular structures.

The magnetic resonance imaging findings of vestibular schwannomas are well known, specific and are mostly intense to pons on MR T1 - weighted images, mildly hyperintense on magnetic resonance T2 - weighted images, and show intense enhancement after I.V. administration of gadolinium DTPA. Homogenous enhancement pattern is noted in cases with meningioma on contrast administration. Epidermoid cysts show no enhancement on magnetic resonance imaging. Early diagnosis helps in the preservation of hearing postsurgery. Also it decreases the rate of complications after surgery.

The results of the present study showed various cerebellopontine angle lesions most commonly encountered in our hospital and the differential diagnostic features of the most important cerebellopontine angle lesions by using radiologic-pathologic correlation. In this study, out of the 50 patients with clinical suspicion of cerebellopontine angle lesions, 22 patients (44\%) had acoustic schwannoma, 14 patients (28\%) had cerebellopontine angle meningioma, 8 patients (16\%) had epidermoid cyst, 3(\%) patients had arachnoid cyst and $3(\%)$ patients were diagnosed to have lipoma and metastasis respectively on MRI. These results are in concordance with the study conducted by Haque $\mathrm{S}$ et al which states that acoustic schwannomas account for nearly $70-80 \%$ of all cerebellopontine angle lesions, followed next in frequency by meningioma and epidermoid cysts in the second and third position constituting $10-15 \%$ and $5 \%$ of cerebellopontine angle tumours respectively. ${ }^{13}$

The age of the patients in the present study ranged from 8 to 72 years with mean age of $51.9+/-10.5$ years which is in concordance to the study done by Mulkens and Osborn et al in which it was found that age range and mean range was 26 - 80 years and 56.5 years respectively. Maximum number of cases were recorded in the age group of 51-60 years which is in accordance to previous studies stating that majority of cases are seen in people post 50 years of life. ${ }^{14,15}$

Out of the 50 patients with clinical suspicion of cerebellopontine angle lesions, 28 patients (56\%) were females and the rest 22 (44\%) were males which agree with the literature affirming that the condition is more common in females comparatively. The study assesses the effectiveness of MRI in the evaluation of cerebellopontine angle lesions in comparison with histopathology as gold standard. MRI showed sensitivity of $95.65 \%$, specificity of $75 \%$ and diagnostic accuracy of $85.33 \%$ while the positive predictive value was 97.78 and its negative predictive value was 60 . These findings of our study are in concordance to previous study done by Swieszewska et al in which magnetic resonance imaging has a sensitivity of $96 \%$, specificity of $88.2 \%$, diagnostic accuracy of 92.86 and positive and predictive value as 92.31 and 93.75 respectively.

Therefore, MRI has greater sensitivity, specificity and diagnostic accuracy and hence gives us a reliable diagnosis, proving to be one of the best imaging modality in the evaluation of cerebellopontine angle lesions. Addition of contrast helped in much better visualization of smaller lesions, which is in consistent to a study done by Swieszewska and Stuckey et al which stated that contrast enhanced T1 - Weighted Magnetic resonance imaging enhances the capacity to visualize the tumour margins and its intrameatal component. ${ }^{16,17}$

Thus, magnetic resonance imaging is a very efficient tool and can be considered as an excellent non-invasive investigation for the evaluation of cerebellopontine angle lesions. It also helps in guiding surgical biopsies thereby decreasing the need for unnecessary intervention. It helps in better detection and characterization of very small lesions as well and also plays an important role in earlier detection, thereby stratifying patients into appropriate treatment options and in assessing the prognosis.

\section{CONCLUSION}

Magnetic Resonance Imaging has excellent diagnostic accuracy and greater sensitivity and specificity in the diagnosis of Cerebellopontine angle lesions and hence can be considered as the imaging modality of choice in the evaluation of CPA tumours.

\section{REFERENCES}

1. Tanweer Ahmad, Noreen Kanwal, Saima Ameer, Nasir S. M. Diagnostic Accuracy of Magnetic Resonance Imaging in detection of acoustic neuroma. Biomedica 2014; 30 (1):17-21.

2. Kunwarpal Singh, Mohit Preet Singh, CL. Thukral, Kiran Rao, Kulvinder Singh, Amandeep Singh. Role of Magnetic Resonance Imaging in Evaluation of Cerebellopontine Angle Schwannomas. Indian J Otolaryngol Head Neck Surg. 2015; 67(1): 21-27.

3. Pustthay Sunil Kumar, P. Sree Hari. Role of MRI in primary malignant bone tumours. International Journal of Contemporary Medical Research 2016; 3(7):21442148.

4. Saravanan K, E.A. Parthasarathy, Abubacker Sulaiman Farook, Praveen Sridharan, Gopalakrishnan, Rajamani Anand. Role of Susceptibility Weighted Imaging in Cerebellopontine Angle Schwannoma v/s Meningioma. International Journal of Contemporary Medicine Surgery and Radiology. 2018; 3(2):B20-B23.

5. Maheswararao Y.V.N, Rama Krishna Rao Baru. MRI evaluation of extra axial cerebello pontine angle tumours. International Journal of Contemporary Medicine Surgery and Radiology. 2018; 3(3):C33- C38.

6. Hart RG, Gardner DP, Howieson J. Acoustic tumours: atypical features and recent diagnostic tests. Neurology 1983; 33(2): 211-221.

7. Clemis JD, Ballad WJ, Baggot PJ, Lyon ST. Relative frequency of inferior vestibular schwannoma. Arch Otolaryngol Head Neck Surg 1986; 112(2):190-194.

8. Joarder MA et al. Surgical outcomes of Cerebellopontine 
angle tumours in 34 cases. Pulse 2015;8(1):8-14.

9. Komatsuzaki A,Tsunoda A. Nerve origin of the acoustic neuroma. J Laryngol Otol 2001; 115(5):376-379.

10. Beaman FD, Kransdorf MJ, Menke DM Schwannoma: radiologic-pathologic correlation. Radiographics 2004; 24(5):1477-1481.

11. Moffat DA, Ballagh RH. Rare tumours of the cerebellopontine angle. Clin Oncol 1995; 7(6):28-41.

12. Bonneville F, Savatovsky J, Chiras J. Imaging of the cerebellopontine angle lesions: an update. J Eur Radiol. 2007; 17(11):2908-2920.

13. Haque S, Hossain A, Quddus MA, Jahan MU. Role of MRI in evaluation of acoustic schwannoma and its comparison to histopathological findings. Bangladesh Med Res Coun Bull 2011;37(3): 92-96.

14. Mulkens TH, Parizel PM, Martin JJ et al. Acoustic schwannoma: MR findings in 84 tumors. AJR. 1993; 160:395-398.

15. Osborn AG. Miscellaneous Tumors, cyst and metastases in diagnostic neuroradiology, Elsevier Publishing, India, 1994; 626-633.

16. Swieszewska, Ewa Izycka, Szurowska, Edyta, Kloc, Wojciech, Rzepko, Rubert, Wybieralska, Mirostawa, Dubaniewicz \& Skurek, Andzej. Cerebello pontine angle tumors radiologic-pathologic correlation. Folia Neuropathol 2006; 44(4): 274-281.

17. Stuckey SL, Harries AJ, Mannolini M. Detection of Acoustic Schwannoma: Use of Constructive Interference in the steady state three dimensional MR. American Society of Neuro Radiology 1996; 17(2): 1219- 1225.

Source of Support: Nil; Conflict of Interest: None

Submitted: 12-06-2019; Accepted: 22-03-2019; Published online: 06-08-2019 